\title{
Effective-particle approach to bound states of quarks and gluons in QCD
}

\author{
María Gómez-Rocha* \\ European Centre for Theoretical Studies in Nuclear Physics and Related Areas (ECT*) \\ E-mail: mariagomezrocha@gmail.com
}

\section{Kamil Serafin ${ }^{\dagger}$}

University of Warsaw - Faculty of Physics

E-mail: Kamil.Serafin@fuw.edu.pl

\begin{abstract}
A general approach to the construction of bound states in quantum field theory, called the renormalization group procedure for effective particles (RGPEP), was applied recently to single heavyflavor QCD in order to study its utility beyond illustration of its general features. This heavy-flavor QCD is chosen as the simplest available context in which the dynamics of quark and gluon bound states can be studied with the required rigor using Minkowski-space Hamiltonian operators in the Fock space, taking the advantage of asymptotic freedom. The effective quarks and gluons differ from the point-like canonical ones by having a finite size $s$. Their size plays the role of renormalization group parameter. However, instead of integrating out high-energy degrees of freedom, our RGPEP procedure is based on a transformation of the front-form QCD Hamiltonian from its canonical form with counterterms to the renormalized, scale-dependent operator that acts in the Fock space of effective quanta of quark and gluon fields, keeping all degrees of freedom intact but accounting for them in a transformed form. We discuss different behavior of effective particles interacting at different energy scales, corresponding to different size s. Namely, we cover phenomena ranging from asymptotic freedom at highest energies down to the scales at which the formation of bound states occurs. We briefly present recent applications of the RGPEP to quarks and gluons in QCD, which have been developed using expansion in powers of the Fock-space Hamiltonian running coupling. After observing that the QCD effective Hamiltonian satisfies the requirement of producing asymptotic freedom, we derive the leading effective interaction between quarks in heavy-flavor QCD. An effective confining effect is derived as a result of assuming that the non-Abelian and non-perturbative dynamics causes effective gluons to have mass.
\end{abstract}

XVII International Conference on Hadron Spectroscopy and Structure - Hadron2017

25-29 September, 2017

University of Salamanca, Salamanca, Spain

\footnotetext{
* Speaker, oral contribution

${ }^{\dagger}$ Speaker, poster contribution
} 


\section{Introduction}

In spite of decades of research the bound-state equation in quantum chromodynamics remains to be an exhausting problem without an exact solution. The elements that build the Schrödingerlike equation in $\mathrm{QCD}, \hat{H}|\psi\rangle=E|\psi\rangle$, which gives hadron masses and wave functions in the Fock space are full of complexities. It is not straightforward how to determine the Hamiltonian whose eigenvalues correspond to hadron masses starting from the QCD Lagrangian density of the theory and using first principles.

The main source of difficulties concerns the fact that in quantum field theory one is forced to deal with an infinite number of degrees of freedom. For instance, a heavy-quarkonium state - the simplest possible system one may consider in QCD - has the structure of an infinite series of Fock components

$$
|\psi\rangle=|Q Q\rangle+|Q Q G\rangle+|Q Q G G\rangle+\ldots,
$$

and in principle, there is no limit on the number of particles allowed.

The renormalization group procedure for effective particles (RGPEP) was formulated as a nonperturbative tool for constructing bound states in QFT. The method stems from the similarity renormalization group (SRG) for Hamiltonians $[1,2]$ and introduces the concept of effective particles. It provides a framework for the description of the interaction of particles at different energy scales. The main idea of the RGPEP is that it is possible to relate the canonical Hamiltonian operator obtained from QCD with an effective one by means of a similarity transformation. The Hamiltonian is written in a scale-dependent operator basis which is such that, for a certain scale, the number of nonnegligible Fock components in the description of hadrons is small. When an infinite number of terms can be neglected in Eq. (1.1), the bound-state problem is thus drastically simplified and one can attempt to seek numerical solutions to the bound-state equation.

In this paper, we summarize the most important new results in the application of the RGPEP to QCD [3,4]. We start presenting the main elements of the RGPEP method in the next section. The procedure is general enough and can be applied to any theory. Afterwards, in Section 3we consider QCD and show how the property of asymptotic freedom arises in the formalism. In Section 4, we consider a theory of only one heavy flavor in QCD and use asymptotic freedom to formulate the bound-state equation for heavy quarkonium. We study the confining properties of the effective potential obtained in the bound-state equation in Section 5. Section 6 concludes the article.

\section{RGPEP}

\subsection{Initial Hamiltonian}

The starting point of our formulation of the bound-state equation is the classical Lagrangian density of the theory. In this work, we are concerned with the Lagrangian of QCD. We use the front form (FF) of Hamiltonian dynamics [5], often called light-front dynamics. ${ }^{1}$ The canonical FF Hamiltonian is obtained by integrating the +- component of the energy momentum tensor $T^{\mu v}$

\footnotetext{
${ }^{1}$ We adopt the notation and conventions given in [6]. The FF coordinates are defined as $x^{\mu}=\left(x^{+}=x^{0}+x^{3}, x^{-}=\right.$ $\left.x^{+}-x^{3}, \vec{x}^{\perp}\right)$, with $x^{\perp}=\left(x^{1}, x^{2}\right)$.
} 
over the quantization surface $x^{+}=0$ in the gauge $A^{+}=0$, and replacing the classical fields by quantum ones $i^{2}$ :

$$
H_{\text {can }}=P^{-}=\left.\frac{1}{2} \int d x^{-} d^{2} x^{\perp} T^{+-}\right|_{x^{+}=0} .
$$

The FF canonical Hamiltonian gives divergent integrals in perturbation theory and needs to be regularized. We introduce two regularization parameters, which regulate interaction vertices: $\Delta$, which regulates ultraviolet divergences related to big changes of perpendicular momentum components $k^{\perp}$; and $\delta$, which regulates the so-called small- $x$ divergences, related to $k^{+}$component of the momentum.

\subsection{The RGPEP equation}

The RGPEP introduces the concept of effective particles of size $s$. The size $s$ is the renormalizationgroup parameter. Creation and annihilation operators labeled by $s$ create or annihilate effective particles of size $s$ :

$$
Q_{0}^{\dagger}|0\rangle=\left|Q_{0}\right\rangle, \quad Q_{0}|0\rangle=0, \quad Q_{s}^{\dagger}|0\rangle=\left|Q_{s}\right\rangle, \quad Q_{s}|0\rangle=0 .
$$

Effective particles of finite size $s$ and bare or canonical ones of size 0 are related by a unitary transformation

$$
Q_{s}=\mathscr{U}_{s} Q_{0} \mathscr{U}_{s}^{\dagger}
$$

For later convenience we define $t=s^{4}$, and express the Hamiltonian in the new basis

$$
H_{t}\left(Q_{t}\right)=H_{0}\left(Q_{0}\right),
$$

where $H_{t}\left(Q_{t}\right)$ means that the Hamiltonian is expressed in terms of effective operators $Q_{t}$ with effective coefficients standing in front of them. The dependence of these coefficients with scale $t$ is given by the equation

$$
\mathscr{H}_{t}=\left[\left[\mathscr{H}_{f}, \mathscr{H}_{P t}\right], \mathscr{H}_{t}\right]
$$

where $\mathscr{H}_{t}=H_{t}\left(Q_{0}\right), \mathscr{H}_{f}$ is the free part of $\mathscr{H}_{t}$, and $\mathscr{H}_{P t}$ is the same as $\mathscr{H}_{t}$ but multiplied by total +-component of momentum entering the vertex squared.

Although Eq. (2.5) can be solved nonperturbatively, at this stage and for the purposes of our current studies, we focus on perturbative solutions to Eq. (2.5),

$$
H_{t}=H_{t 0}+g H_{t 1}+g^{2} H_{t 2}+g^{3} H_{t 3}+g^{4} H_{t 4}+\ldots
$$

Solving Eq. (2.5) order by order yields exponentials $\exp \left[-t\left(\mathscr{M}_{c}^{2}-\mathscr{M}_{a}^{2}\right)^{2}\right]$, where $\mathscr{M}_{c}$ and $\mathscr{M}_{a}$ are invariant masses of particles created and annihilated in a vertex, which play the role of

\footnotetext{
${ }^{2}$ We use the usual Fourier decomposition,

$\hat{A}^{\mu}=\sum_{\sigma c} \int[k]\left[t^{c} \varepsilon_{k \sigma}^{\mu} \hat{a}_{k \sigma c} e^{-i k x}+t^{c} \varepsilon_{k \sigma}^{\mu *} \hat{a}_{k \sigma c}^{\dagger} e^{i k x}\right]_{x^{+}=0}, \quad \hat{\psi}=\sum_{\sigma c f} \int[k]\left[\chi_{c} u_{f k \sigma} \hat{b}_{k \sigma c f} e^{-i k x}+\chi_{c} v_{f k \sigma} \hat{d}_{k \sigma c f}^{\dagger} e^{i k x}\right]_{x^{+}=0}$.
} 
form factors appearing at interacting vertices [7]. The effective Hamiltonian is determined by the initial condition that at $t=0$ the Hamiltonian must equal the regularized canonical Hamiltonian plus counterterms. The counterterms are determined by the condition that every matrix element of the effective theory $(t>0)$ is cutoff independent when the ultraviolet regularization is removed.

The notion of effective particles can be also understood using the parameter $\lambda=1 / \mathrm{s}$ which has dimension of mass. Namely, due to form factors, effective particles of type $\lambda$ cannot change their relative kinetic energy through a single effective interaction by more that about $\lambda$.

\section{Asymptotic Freedom}

The first check that was made in the application of the RGPEP to QCD was its suitability to reproduce the property of asymptotic freedom [3] and the agreement of the result with the one obtained using another generator [8]. The feature of asymptotic freedom at short distances could be checked in terms of a family of renormalized effective Hamiltonians using RGPEP. The structure of the three-gluon and quark-gluon vertices can be extracted from third-order solutions to the RGPEP equation for QCD. It was shown in Ref. [3] that for a quantum Yang-Mills theory the Hamiltonian running coupling evolves with the scale as

$$
g_{\lambda}=g_{0}-\frac{g_{0}^{3}}{48 \pi^{2}} N_{c} 11 \ln \frac{\lambda}{\lambda_{0}},
$$

which agrees with the known function obtained in $[9,10]$. Whereas the running coupling described using Feynman diagrams evolves with momentum scale, the Hamiltonian running coupling obtained within RGPEP evolves with the parameter $\lambda$.

We will make use of the property of asymptotic freedom to derive an effective theory of heavy quarks in the next section.

\section{Heavy quarkonium problem}

The simplest bound system one can consider in QCD is heavy quarkonium. To simplify the picture, in the following, we consider only one flavor of heavy quarks and neglect light quarks. The eigenproblem may be simplified drastically with the following choice of the renormalization group parameter,

$$
m \gg \lambda \gg \Lambda_{\mathrm{QCD}}
$$

where $m$ is the quark mass. On the one hand, $\lambda \gg \Lambda_{\mathrm{QCD}}$ allows us to expand the Hamiltonian in powers of $g_{t}$ and keep only the first few terms due to asymptotic freedom. On the other hand, because $m \gg \lambda$, Fock sectors with extra quark-antiquark pairs are strongly suppressed by RGPEP form factors and we may neglect them. Sectors with gluons cannot be a priori neglected, because gluons are massless and one may produce many of them without adding much to the invariant mass of a system (contrary to the addition of heavy quarks). This poses a problem, beacause one cannot deal with infinitely many Fock sectors. The solution we adopt is that we drop all the sectors with two or more gluons, but make up for their absence by introducing a gluon mass ansatz in the sector $Q_{t} \bar{Q}_{t} G_{t}$. The gluon mass ansatz is motivated by three observations. First, it is the simplest term one 
can add. Second, an effective gluon mass might arise as a nonperturbative effect when the reduction of the Fock space is done exactly. This effect contains nonabelian gluon-gluon interactions. Third, the phenomenology of hadrons seems to exclude massless effective gluons.

The heavy quarkonium bound-state problem with just two Fock sectors and gluon mass ansatz is (see Ref. [4] for more details)

$$
\left[\begin{array}{ll}
H_{f}+\mu^{2} & g H_{t 1} \\
g H_{t 1} & H_{f}+g^{2} H_{t 2}
\end{array}\right]\left[\begin{array}{l}
\left|Q_{t} \bar{Q}_{t} G_{t}\right\rangle \\
\left|Q_{t} \bar{Q}_{t}\right\rangle
\end{array}\right]=E\left[\begin{array}{l}
\left|Q_{t} \bar{Q}_{t} G_{t}\right\rangle \\
\left|Q_{t} \bar{Q}_{t}\right\rangle
\end{array}\right],
$$

where $\mu^{2}$ is the gluon-mass-like operator acting within the $Q_{t} \bar{Q}_{t} G_{t}$ sector. An important assumption about $\mu^{2}$ is that it depends on the relative motion of the gluon with respect to the quark-antiquark pair in $Q_{t} \bar{Q}_{t} G_{t}$ sector. The need for this dependence is briefly explained in Sec. 5 .

Because we kept only terms order $1, g$ and $g^{2}$ in the approximate eigenvalue problem Eq. (4.2), we may perturbatively eliminate the sector with gluon reducing the eigenproblem to the lowest sector [11]. Matrix elements of the effective Hamiltonian obtained as a result of this elimination are

$$
\left\langle l\left|H_{t \mathrm{eff}}\right| r\right\rangle=\left\langle l\left|\left\{H_{f}+g^{2} H_{t 2}+\frac{1}{2} g H_{t 1}\left[\frac{1}{E_{l}-H_{f}-\mu^{2}}+\frac{1}{E_{r}-H_{f}-\mu^{2}}\right] g H_{t 1}\right\}\right| r\right\rangle,
$$

where $|l\rangle$ and $|r\rangle$ are both in $Q_{t} \bar{Q}_{t}$ sector and $H_{f}|l\rangle=E_{l}|l\rangle$ and $H_{f}|r\rangle=E_{r}|r\rangle$.

\section{Result: Coulomb and harmonic oscillator potentials}

As a result of elimination of the sector containing one gluon we obtain a Hamiltonian acting in the lowest sector, $Q \bar{Q}$. The FF eigenvalue equation is

$$
H_{t \mathrm{eff}}\left|\psi_{Q \bar{Q} t}\right\rangle=\frac{M^{2}+P^{\perp 2}}{P^{+}}\left|\psi_{Q \bar{Q} t}\right\rangle
$$

where $M$ is the mass while $P^{+}$and $P^{\perp}$ are longitudinal and perpendicular momenta of the state

$$
\left|\psi_{Q \bar{Q} t}\right\rangle=\sum_{24} P^{+} \frac{\delta_{c_{2} c_{4}}}{\sqrt{3}} \tilde{\delta}\left(P-k_{2}-k_{4}\right) \psi_{t 24}\left(\kappa_{24}^{\perp}, x_{2}\right) b_{2 t}^{\dagger} d_{4 t}^{\dagger}|0\rangle .
$$

$\delta_{c_{2} c_{4}} / \sqrt{3}$ is the color singlet wave function, $\tilde{\delta}$ is a momentum conservation Dirac delta multiplied by $16 \pi^{3}$ and $\psi_{t 24}\left(\kappa_{24}^{\perp}, x_{2}\right)$ is a FF wave function depending on spins (indicated by subscripts 24 , cf. Figure 1), relative $\perp$-momentum $\kappa_{24}^{\perp}=x_{4} k_{2}-x_{2} k_{4}$, and longitudinal momentum fraction $x_{i}=$ $k_{i}^{+} / P^{+}$carried by the particle $i$. It is a property of the front form that relative motion described by momenta $\kappa_{i j}^{\perp}$ and $x_{i}$ decouples from the absolute motion with momenta $P^{+}$and $P^{\perp}$. Therefore, the FF eigenvalues are in fact masses squared $M^{2}$. Equation (5.1) is rewritten in terms of the FF wave function,

$$
\left(\frac{\mathcal{M}_{t}^{2}+\kappa_{13}^{\perp 2}}{x_{1} x_{3}}-M^{2}\right) \psi_{t 13}\left(\kappa_{13}^{\perp}, x_{1}\right)+g^{2} \int \frac{d x_{2} d^{2} \kappa_{24}^{\perp}}{2(2 \pi)^{3} x_{2} x_{4}} U_{t \mathrm{eff}}(13,24) \psi_{t 24}\left(\kappa_{24}^{\perp}, x_{2}\right)=0 .
$$

$\mathcal{M}_{t}^{2}=m^{2}+\delta m_{t}^{2}$ is the quark mass squared plus the second order effective quark self-interaction term, $U_{t \text { eff }}(13,24)$ is the effective interaction between quark and antiquark coming from exchanging gluons. 

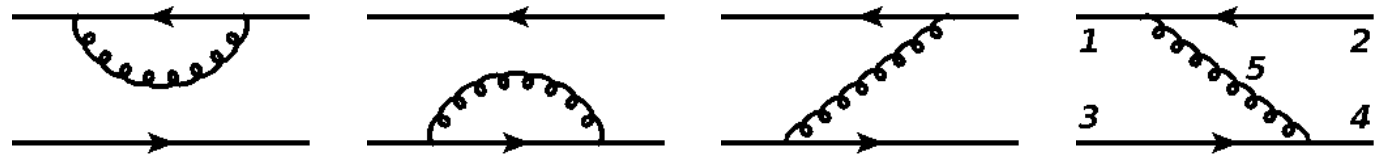

Figure 1: Self-interaction terms and gluon exchange terms in Eq. (5.3).

Equation (5.3) with large relative momenta between quarks suppressed by form factors due to $\lambda \ll m$ and with small coupling constant due to $\lambda \gg \Lambda_{\mathrm{QCD}}$ may be approximated by its nonrelativistic limit. However, before looking for approximations we need to check if all regularization dependence of Eq. (5.3) is removed. The problem is that there might appear small-x divergences when we add an ansatz. For example, $\delta m_{t}^{2}$, which is zero in the absence of gluon mass ansatz, is potentially divergent when small-x regularization is lifted. Similar divergences are present also in the exchange terms. To avoid any regularization dependence we have to assume that $\mu^{2}$ vanishes in a proper way when $x_{5} \rightarrow 0$. For example, $\mu^{2} \sim x_{5}^{\delta_{\mu}} \kappa_{5}^{2}$ when $x_{5} \rightarrow 0$ (where $x_{5}$ and $\kappa_{5}^{\perp}$ are relative FF momenta of gluon with respect to the $Q \bar{Q}$ pair) with $0<\delta_{\mu}<1 / 2$ is sufficient to guarantee that the effective Hamiltonian is finite when the regularization is removed. The validity of the gluon mass ansatz may be checked by performing 4th order calculation of the effective Hamiltonian for quarkonium. However, details of the gluon mass ansatz turn out not to be important for the main result, Eq. (5.8).

To write the nonrelativistic approximation of Eq. (5.3) we need momentum variables, which are more suitable than $\kappa^{\perp}$ and $x$. We define

$$
k_{i j}^{\perp}=\frac{1}{2} \frac{\kappa_{i j}^{\perp}}{\sqrt{x_{i} x_{j}}}, \quad k_{i j}^{3}=\frac{m}{\sqrt{x_{i} x_{j}}}\left(x_{i}-\frac{1}{2}\right),
$$

where $i j=13$ or $i j=24$, according to Fig. 1 . We define also $M=2 m+B$, where $B$ is binding energy, divide both sides of Eq. (5.3) by $4 m$, and take the limit $\vec{k}_{i j} / m \rightarrow 0$. The result is

$$
\left[\frac{\vec{k}_{13}^{2}}{m}-B+\frac{\delta m_{t}^{2}}{m}\right] \psi_{13}\left(\vec{k}_{13}\right)+\int \frac{d^{3} q}{(2 \pi)^{3}}\left[V_{C, B F}+W(\vec{q})\right] \psi_{24}\left(\vec{k}_{13}-\vec{q}\right)=0,
$$

where $V_{C, B F}$ stands for Coulomb potential with Breit-Fermi spin-dependent interactions,

$$
V_{C, B F}=-\frac{4}{3} \frac{4 \pi \alpha}{\vec{q}^{2}}(1+B F) e^{-16 s^{4}\left(k_{13}^{2}-k_{24}^{2}\right)^{2}},
$$

$4 \pi \alpha=g^{2}, \vec{q}=\vec{k}_{13}-\vec{k}_{24}$, and

$$
W(\vec{q})=-\frac{4}{3} 4 \pi \alpha\left(\frac{1}{q_{z}^{2}}-\frac{1}{\vec{q}^{2}}\right) \frac{\mu^{2}}{\mu^{2}+\vec{q}^{2}} e^{-2 t m^{2} \frac{q^{4}}{q_{z}^{2}}} .
$$

The exponential factor limits $q$ to small values for $\lambda \ll m$, hence $\mu^{2} /\left(\mu^{2}+q^{2}\right) \rightarrow 1$ as long as $\mu \neq 0$ and we can expand the wave function $\psi_{24}\left(\vec{k}_{13}-\vec{q}\right)$ at $\vec{q}=0$. The first term (independent of $\vec{q}$ ) cancels with the mass term $\delta m_{t}^{2} / m=-\int d^{3} q W(\vec{q}) /(2 \pi)^{3}$, while the terms linear in $\vec{q}$ vanish when integrated over $\vec{q}$. Terms quadratic in $\vec{q}$ produce a correction to Coulomb interaction proportional to $-\Delta_{\vec{k}} \psi=r^{2} \psi$, where $r$ is the distance between quark and antiquark. The final result is

$$
\frac{\vec{k}_{13}^{2}}{m} \psi_{13}\left(\vec{k}_{13}\right)+\int \frac{d^{3} q}{(2 \pi)^{3}} V_{C, B F} \psi_{24}\left(\vec{k}_{13}-\vec{q}\right)+\frac{1}{2} \frac{m}{2} \omega^{2} r^{2} \psi_{13}\left(\vec{k}_{13}\right)=B \psi_{13}\left(\vec{k}_{13}\right)
$$


where the harmonic oscillator frequency is $\omega=\sqrt{\frac{\alpha}{18 \sqrt{2 \pi}}} \frac{\lambda^{3}}{m^{2}}$.

\section{Conclusion}

The RGPEP is a tool suitable for the task of calculating bound states in QCD. It passes the test of producing asymptotic freedom in the running of the effective coupling constant and in the second order calculation with a gluon mass ansatz it produces a Schrödinger equation for heavy quarks with Coulomb and harmonic oscillator potentials. Harmonic oscillator potential in front form corresponds to linear potential in the usual equal-time formulation [12]. Therefore, it is expected to be a good first approximation for calculations of hadron spectra. Moreover, the same harmonic oscillator potential was found using a different version of RGPEP [13], establishing a welcome degree of universality of the harmonic oscillator potential result. Although this result relies on the ansatz, the method presented here can be extended to $g^{4}$ order and to include $Q_{t} \bar{Q}_{t} G_{t} G_{t}$ sector. In such calculation, the gluon mass ansatz from $Q_{t} \bar{Q}_{t} G_{t}$ sector will be replaced by true QCD and the ansatz will be verified. Another research goal is a nonperturbative determination of running of effective quark and gluon masses with renormalization scale $\lambda$.

\section{References}

[1] S. D. Glazek and K. G. Wilson, Renormalization of Hamiltonians, Phys. Rev. D48 (1993) 5863-5872.

[2] K. G. Wilson, T. S. Walhout, A. Harindranath, W.-M. Zhang, R. J. Perry and S. D. Glazek, Nonperturbative QCD: A Weak coupling treatment on the light front, Phys. Rev. D49 (1994) 6720-6766, [hep-th/9401153].

[3] M. Gómez-Rocha and S. D. Głazek, Asymptotic freedom in the front-form Hamiltonian for quantum chromodynamics of gluons, Phys. Rev. D92 (2015) 065005, [1505.06688].

[4] S. D. Głazek, M. Gómez-Rocha, J. More and K. Serafin, Renormalized quark-antiquark Hamiltonian induced by a gluon mass ansatz in heavy-flavor QCD, Phys. Lett. B773 (2017) 172-178.

[5] P. A. M. Dirac, Forms of Relativistic Dynamics, Rev. Mod. Phys. 21 (1949) 392-399.

[6] S. J. Brodsky, H.-C. Pauli and S. S. Pinsky, Quantum chromodynamics and other field theories on the light cone, Phys. Rept. 301 (1998) 299-486, [hep-ph/9705477].

[7] S. D. Glazek, Perturbative formulae for relativistic interactions of effective particles, Acta Phys. Polon. B43 (2012) 1843-1862, [120 4 4 760].

[8] S. D. Glazek, Dynamics of effective gluons, Phys. Rev. D63 (2001) 116006, [hep-th/ 0012012 ].

[9] H. D. Politzer, Reliable Perturbative Results for Strong Interactions?, Phys. Rev. Lett. 30 (1973) 1346-1349.

[10] D. J. Gross and F. Wilczek, Ultraviolet Behavior of Nonabelian Gauge Theories, Phys. Rev. Lett. 30 (1973) 1343-1346.

[11] K. G. Wilson, A model of coupling constant renormalization, Phys. Rev. D2 (1970) 1438.

[12] A. P. Trawiński, S. D. Głazek, S. J. Brodsky, G. F. de Téramond and H. G. Dosch, Effective confining potentials for QCD, Phys. Rev. D90 (2014) 074017, [1403.5651].

[13] S. D. Glazek, Harmonic oscillator force between heavy quarks, Phys. Rev. D69 (2004) 065002, [hep-th/0307064]. 